\title{
Glucose transport and sensing in the maintenance of glucose homeostasis and metabolic harmony
}

\author{
Mark A. Herman and Barbara B. Kahn
}

\author{
Division of Endocrinology, Diabetes and Metabolism, Department of Medicine, Beth Israel Deaconess Medical Center and Harvard Medical School, \\ Boston, Massachusetts, USA.
}

\begin{abstract}
Recent data underscore the importance of intertissue communication in the maintenance of normal glucose homeostasis. Important signals are conveyed by hormones, cytokines, and fuel substrates and are sensed through a variety of cellular mechanisms. The ability of tissues to sense and adapt to changes in metabolic status and fuel availability is altered in insulin-resistant states including type 2 diabetes. Here we review the roles of glucose and its metabolites as signaling molecules and the diverse physiologic mechanisms for glucose sensing.
\end{abstract}

\section{Introduction}

The prevalence of obesity and type 2 diabetes continues to increase at alarming rates (1). Type 2 diabetes is a prototypic complex, polygenic disease with a strong heritable component, which is also heavily influenced by environmental factors - especially diet and physical activity. It appears that altered communication among tissues and loss of the ability of tissues to adapt to changing metabolic states play a critical role in the altered glucose homeostasis that leads to the development of type 2 diabetes. As McGarry illuminated, the utilization of many fuels, particularly lipids, is also altered in diabetes (2). However, this review will focus on the role of glucose and its metabolites as signals underlying the highly orchestrated "intertissue communication" that regulates glucose homeostasis.

Overt diabetes mellitus is defined clinically by fasting or postprandial hyperglycemia or an abnormally increased glucose excursion in response to a defined glucose load. However, this clinical definition defines a relatively late stage in the disease process. Significant defects in glucose homeostasis and fuel metabolism are detectable long before overt diabetes occurs. Insulin resistance, measured as impaired glucose disposal in a hyperinsulinemic-euglycemic clamp study, is one of the earliest detectable derangements and is considered a cardinal pathophysiologic feature (3). Fasting hyperinsulinemia is also present early in the disease process and is thought to be a compensatory mechanism to maintain euglycemia in the setting of insulin resistance (4). Overt diabetes mellitus occurs when pancreatic $\beta$ cells can no longer secrete sufficient insulin to compensate for insulin resistance.

Kelley and colleagues have recently proposed a new central pathophysiologic construct to describe the altered metabolism associated with insulin-resistant and glucose-intolerant states: the concept of "metabolic inflexibility" (5). Metabolically normal people can adapt to the discontinuities in fuel availability and utilization present in daily life, whereas diabetic people cannot. Metabolic inflexibility means that insulin-resistant individuals

Nonstandard abbreviations used: AG4KO, adipose-specific GLUT4-knockout; AMPK, AMP-activated protein kinase; ChREBP, carbohydrate response element-binding protein; GFAT, glutamine:fructose-6-phosphate amidotransferase; GLUT, facilitated-diffusion glucose/hexose transporter; GSIS, glucose-stimulated insulin secretion; $K_{A T P}$ channel, ATP-sensitive potassium channel; MG4KO, muscle-specific GLUT4-knockout; PEPCK, phosphoenolpyruvate carboxykinase; PGC-1 $\alpha, \operatorname{PPAR} \gamma$, coactivator 1- $\alpha$; RBP4, retinol-binding protein 4; SIRT1, sirtuin 1.

Conflict of interest: B.B. Kahn has a research grant from Takeda Pharmaceutical Co. Citation for this article: J. Clin. Invest. 116:1767-1775 (2006). doi:10.1172/JCI29027. are unable to efficiently increase carbohydrate utilization, even when carbohydrates are plentiful. This, in some sense, is a restatement of the essence of impaired glucose tolerance (and insulin resistance). However, insulin-resistant people also do not appropriately increase fatty acid oxidation during fasting (6) but remain locked in a middle ground between a carbohydrate and fatty acid fuel economy regardless of fuel availability.

Integrated fuel homeostasis relies on interactions among numerous tissues to orchestrate the seamless transition between widely varying states, including the fed state, fasting, and exercise. How are the complex subsystems that govern glucose homeostasis in the normal state regulated, and what goes awry to cause the diabetic state? We previously asked the metaphorical question, "Who is conducting the orchestra?" (7). In which tissue does the primary defect reside in insulin-resistant or type 2 diabetic states? Compelling advances over the last 8 years stimulated us to modify the question to "How do the individual instruments make a harmony?" For instance, during a transition from the resting state to exercise, a profound and rapid shift in whole-body fuel metabolism must occur in response to the rapid increase in the metabolic demands of skeletal muscle (reviewed in ref. 8). The increased energy demands in muscle at the onset of exercise presumably generate signals to convey this change in physiology to the rest of the body. The metabolic inflexibility observed in insulin-resistant people involves a loss of adaptability. What has led to the loss of flexibility? Is it a breakdown in communication, and if so, what are the signals that have gone awry? In this review we will emphasize the importance of glucose and glucose-derived metabolites as signals (Figure 1) to maintain normal glucose homeostasis and will indicate, primarily using transgenic mouse models, how alterations in these pathways may perturb glucose homeostasis.

\section{Lessons from genetic modifications of glucose transport} Given the fact that glucose transport is the rate-limiting step in insulin-stimulated glucose disposal in muscle and adipocytes, several laboratories have undertaken studies over the last 2 decades to determine the physiologic relevance of glucose transport in tissues implicated as key regulators of glucose homeostasis. The development of models with genetic modification of glucose transport in various tissues has led to numerous surprising observations regarding the impact of glucose transport in insulin target tissues on whole-body fuel homeostasis. These studies suggest that glucose sensing is not restricted to the pancreatic $\beta$ cell, neurons, and 


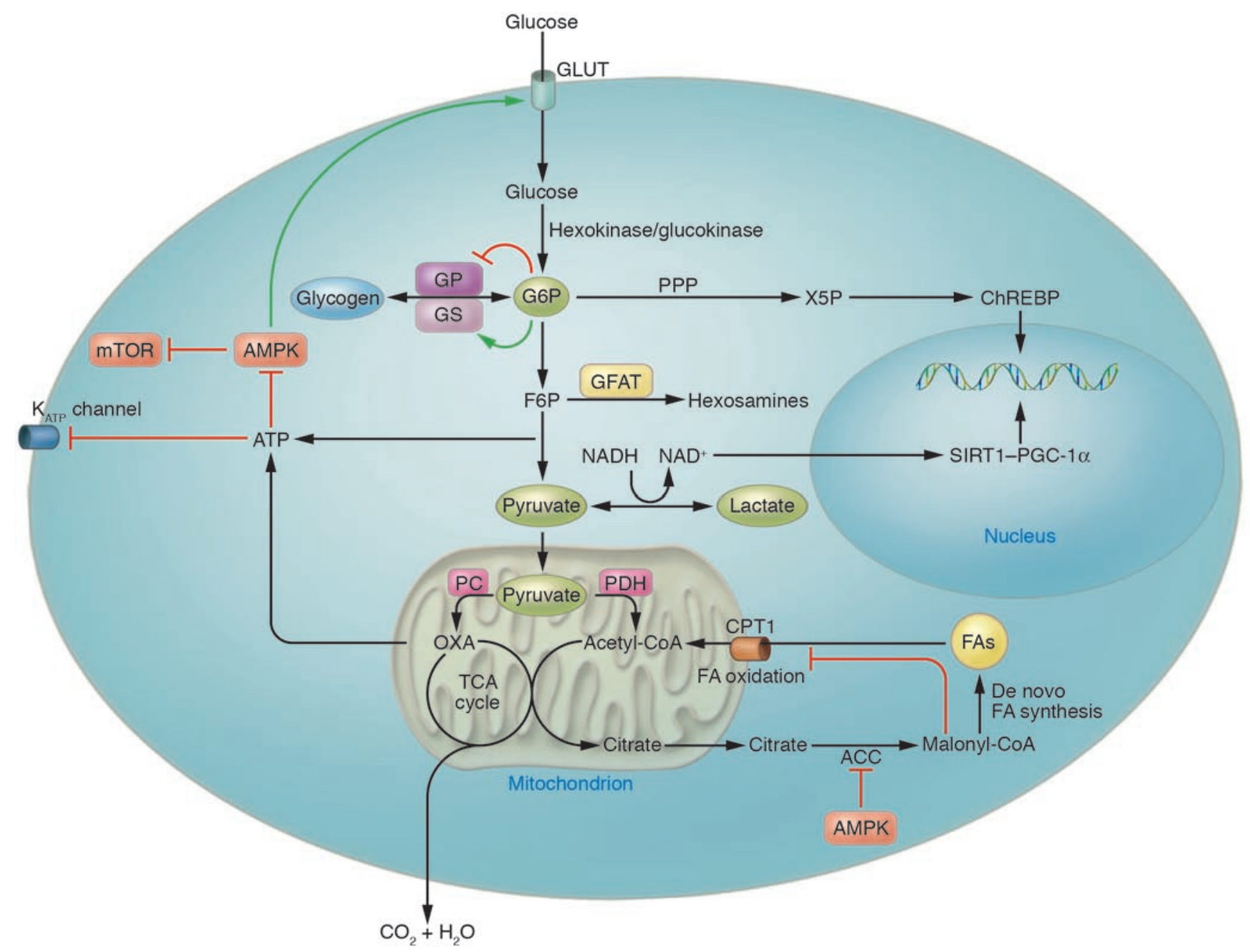

Figure 1

Molecular glucose sensing. Upon entering cells of various types, numerous glucose-derived metabolites are sensed by a variety of cellular sensors, including glycogen synthase, ChREBP, AMPK, SIRT1-PGC-1 $\alpha$, carnitine palmitoyl transferase 1 (CPT1), and $\mathrm{K}_{\text {ATP }}$ channels. Via its diverse metabolites and their sensors, glucose entering the cell changes gene transcription, modulates signal transduction networks, and alters substrate flux through anabolic and catabolic pathways. ACC, acetyl-CoA carboxylase; FA, fatty acid; F6P, fructose-6-phosphate; GLUT, facilitative glucose transporter; GP, glycogen phosphorylase; G6P, glucose-6-phosphate; GS, glycogen synthase; HK/GK, hexokinase/glucokinase; mTOR, mammalian target of rapamycin; OXA, oxaloacetate; PC, pyruvate carboxylase; PDH, pyruvate dehydrogenase; PPP, pentose phosphate pathway.

glial cells and that tissues such as muscle and adipose may also sense glucose and communicate changes in glucose flux to other tissues to impact systemic glucose homeostasis.

The sodium-independent, facilitated-diffusion glucose/hexose transporters (GLUTs) are a family of integral membrane proteins that mediate the transport of hexoses across plasma membranes (3). The members of the GLUT family are distinguished by differential affinities for their substrates and tissue-specific expression and regulation (9).

GLUT4, the major insulin-stimulated glucose transporter, is expressed predominantly in skeletal muscle, cardiac muscle, and adipose tissue and is largely responsible for insulin-stimulated glucose transport into these tissues (3). GLUT4 is sequestered in intracellular vesicles in the absence of insulin. Upon insulin stimulation, GLUT4 vesicles translocate to and fuse with the plasma membrane, permitting increased glucose flux. Glucose transport via GLUT4 into muscle and adipose tissue is the rate-controlling step in insulin-mediated glucose disposal, and this disposal is diminished in insulin-resistant states. GLUT1, which is constitutively present on the plasma membrane and in intracellular membranes, plays a minor role in glucose transport in insulin-responsive tissues, although it plays a major role in other tissues. Important insights into the mechanisms governing glucose homeostasis have been gained from studying the physiological consequences of genetically modifying GLUT expression in specific tissues.

Transgenic overexpression of GLUT1 in skeletal muscle results in a 3- to 4-fold increase in basal glucose uptake in muscle ex vivo (10). GLUT1 overexpression causes fasting hypoglycemia, reduces glycemia in the fed state, and improves glucose tolerance. Plasma lactate and $\beta$-hydroxybutyrate are elevated in both fed and fasted muscle-specific GLUT1 overexpressors. Despite fasting hypoglycemia in these transgenic animals, insulin levels are not lower than 
those in controls, suggesting that altering the flux of glucose into muscle alters the set point for glycemia (10). While the increased plasma lactate may result from increased lactate production by muscle saturated with glucose, the mechanism by which changing glucose flux into muscle alters the set point for basal glycemia is unknown. Despite fasting hypoglycemia and improved glucose tolerance, euglycemic-hyperinsulinemic clamp studies demonstrate insulin resistance in muscle-specific GLUT1-overexpressing mice (11). Consistent with the in vivo findings, insulin does not increase glucose transport in muscle studied ex vivo from these transgenic mice (10).

Similar to the muscle-specific GLUT1 overexpression model, transgenic mice overexpressing GLUT4 in both muscle and adipose tissue also show a marked reduction in fed and fasted glucose levels in addition to improved glucose tolerance (12). Similar to the muscle-specific GLUT1 overexpressors, muscle/fat-specific GLUT4 overexpressors also demonstrate increases in circulating lactate and $\beta$-hydroxybutyrate levels. In contrast to the musclespecific GLUT1 overexpressors, insulin levels are appropriately decreased in response to the basal hypoglycemia present in the muscle/fat-specific GLUT4 overexpressors (12). In addition, the muscle/fat-specific GLUT4 overexpressors demonstrate increased glucose disposal in response to insulin during a clamp, in contrast to the insulin resistance in muscle-specific GLUT1 overexpressors (11). Two separate models with muscle-specific GLUT4 overexpression exhibit phenotypes that are generally consistent with those of the muscle/fat-specific GLUT4 overexpressor, i.e., improved glucose tolerance and insulin responsiveness $(13,14)$. These muscle-specific GLUT4-overexpressing models substantiate the importance of glucose transport in muscle for glucose homeostasis, independent of effects of GLUT4 in adipose tissue.

Transgenic mice with adipose-specific overexpression of GLUT4 show a 2- to 3-fold increase in basal and insulin-stimulated glucose disposal into isolated adipocytes at physiologic glucose concentrations ex vivo (15). Transport of 2-deoxyglucose into adipose tissue in vivo is modestly increased, but, consistent with the notion that adipose tissue accounts for only a small fraction of insulin-stimulated glucose disposal, no difference in insulin-stimulated glucose disposal can be detected at the whole-body level by euglycemichyperinsulinemic clamp studies (16). However, adipose-specific overexpression of GLUT4 leads to a degree of fasting hypoglycemia comparable to that of the muscle-specific GLUT1 overexpressor and the combined muscle/fat-specific GLUT4 overexpressor.

Muscle-specific GLUT4-knockout (MG4KO) mice have a 95\% reduction in skeletal and cardiac muscle GLUT4 protein (17). GLUT1 levels are normal in skeletal muscle but increased approximately 2 -fold in cardiac muscle. Given that skeletal muscle accounts for the majority of glucose disposal during a glucoseinsulin infusion, the development of hyperglycemia, glucose intolerance, and insulin resistance as early as 8 weeks of age in these mice is not surprising. However, it is surprising that insulinstimulated glucose transport in adipose tissue and suppression of hepatic glucose production by insulin are impaired in these mice. Thus, the diminished glucose transport in muscle due to genetic downregulation of GLUT4 selectively in muscle secondarily causes insulin resistance in additional insulin target tissues (18). In this case, hyperglycemia as a direct result of impaired glucose disposal in muscle appears to convey a signal to liver and adipose tissue, resulting in insulin resistance in these tissues, as chronic Phloridzin treatment, which normalizes blood glucose in diabetic animals by increasing glucose excretion from the kidney, normalizes insulin action in adipose tissue and liver of MG4KO mice (18). Interestingly, adipose-specific Glut4 overexpression in MG4KO mice rescues the insulin resistance and glucose intolerance present in MG4KO mice (16). This demonstrates the importance of intertissue communication in glucose homeostasis and the dominant effect that adipocyte-derived signals can have.

In adipose-specific GLUT4-knockout (AG4KO) mice, GLUT4 protein levels are reduced by $70-99 \%$ in both brown and white adipose tissue, with preservation of GLUT4 levels in muscle. These mice have normal adipose mass and normal adipocyte size. Although white adipose tissue accounts for less than $10 \%$ of whole-body glucose uptake in nonobese mice (19), AG4KO mice have insulin resistance and glucose intolerance comparable to those observed in the MG4KO mice. Thus, the reduction in insulin-stimulated glucose transport in adipocytes secondarily induces insulin resistance in other insulin target tissues, though the nature of the signal that mediates this communication is unclear. We recently described a new adipokine, retinol-binding protein 4 (RBP4), which contributes to the insulin resistance that develops in the AG4KO mouse model (20). This model is particularly important since insulin-resistant humans and rodents exhibit downregulation of GLUT4 expression selectively in adipocytes and not in skeletal muscle, although insulin-stimulated glucose transport is also impaired in muscle (3).

It is likely that the physiologic changes brought about by adipose-specific GLUT4 knockout are due to changes in glucose flux although they could result from other unknown consequences of diminished transporter expression. If these changes are the direct result of changes in glucose flux, then adipose tissue may have the capacity for glucose sensing. Is glucose sensing distributed across diverse tissue types, and if so, how are changes in glucose flux interpreted and communicated to other tissues?

With the recent identification of multiple novel hormones implicated in glucose homeostasis, intertissue communication has emerged as a central theme in the study of normal and pathologic fuel physiology. The signals that connect diverse tissues can take many forms, including circulating hormones and cytokines and neural pathways. In addition, circulating energetic substrates like fatty acids and amino acids, which may be directly affected by changes in the insulin-glucose axis, may also act as signaling molecules. For instance, an insulin-glucose infusion in the setting of a euglycemic-hyperinsulinemic clamp study reduces circulating leucine levels as a result of insulin-stimulated protein synthesis (21). Leucine has been implicated as a critical signaling molecule for insulin-independent activation of the ubiquitous mammalian target of rapamycin (mTOR) nutrient-sensing pathway $(22,23)$. The signals that convey changes in glucose flux may take many potential forms, and these pathways must be taken into account to understand the mechanisms by which tissue-specific changes in glucose flux alter whole-body glucose homeostasis.

\section{Molecular and cellular glucose sensing}

Glucose-stimulated insulin secretion (GSIS) in the pancreatic $\beta$ cell is the most extensively studied model of cell-autonomous glucose sensing in mammalian systems. Glucose enters the islet $\beta$ cell via facilitated diffusion through GLUT2, the predominant glucose transporter in $\beta$ cells. In pancreatic $\beta$ cells, glucokinase, not glucose transport, is rate limiting for glucose metabolism at physiological glucose concentrations (24). In addition, glucokinase, unlike other hexokinases, is not allosterically inhibited by 


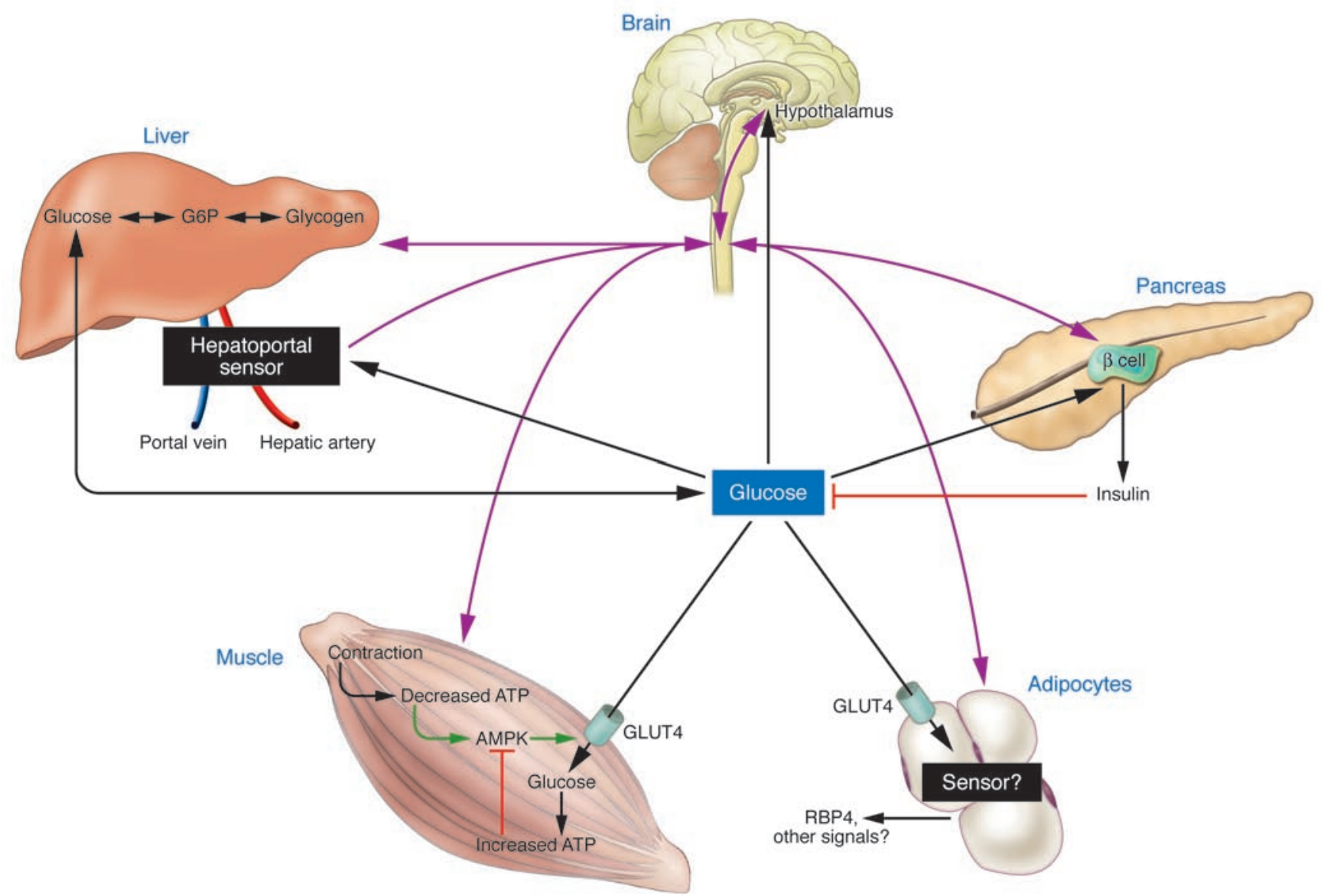

Figure 2

Intertissue glucose sensing and communication. Glucose is sensed in numerous tissues and cell types, including the hypothalamus, hepatocytes, the hepatoportal sensor, pancreatic islets, and possibly muscle and adipose tissue, each of which communicates with other tissues via hormones, neural pathways, or changes in the utilization of substrate. Pink lines represent neural mediated communication. Black lines represent glucose- or hormone-mediated communication.

glucose-6-phosphate. Thus, glycolytic flux is proportional to the extracellular glucose concentration. Increases in the ATP/ADP ratio generated as a result of this glucose flux are thought to lead to $\beta$ cell depolarization via closure of ATP-sensitive potassium channels ( $\mathrm{K}_{\mathrm{ATP}}$ channels) and subsequent insulin secretion (for review, see ref. 25).

However, ATP generation via glycolysis is not the only factor regulating GSIS in the $\beta$ cell. The pancreatic $\beta$ cell is relatively unique in that it expresses low levels of lactate dehydrogenase and high levels of pyruvate carboxylase, an anaplerotic enzyme (26). Anaplerosis refers to biochemical reactions that increase the net carbon content of the TCA cycle (27). Flux through pyruvate carboxylase leads to an accumulation of carbon within the TCA cycle, whereas flux through pyruvate dehydrogenase does not (Figure 1 ). With the latter enzyme, the 2 carbons added to the TCA cycle as acetyl-CoA are fully oxidized to $\mathrm{CO}_{2}$ and $\mathrm{H}_{2} \mathrm{O}$ with one turn of the cycle. As the mitochondrial matrix cannot act as a sink for TCA cycle intermediates, carbon entering the cycle through pyruvate carboxylase must leave the mitochondria as TCA intermediates to maintain a stable, functional level of TCA intermediates. Evidence is accumulating that one or more of these TCA cycle intermediates exported from the mitochondria to maintain bal- ance between anaplerosis and cataplerosis (involving net loss of carbon from the TCA cycle) is likely to serve as a metabolic coupling factor for GSIS (28). Therefore, while glucose plays a role in providing a substrate for energy generation, specific intracellular glucose-derived metabolites appear to have independent roles as signaling molecules (Figure 1).

Malonyl-CoA. Malonyl-CoA derived from citrate exported from the mitochondria is one such potential cataplerotic metabolite implicated in glucose sensing in numerous tissues, including the $\beta$ cell (reviewed in ref. 29). Malonyl-CoA, an intermediate in de novo fatty acid synthesis, is an allosteric inhibitor of carnitine palmitoyltransferase 1 (CPT1), which regulates the transport of long-chain fatty acyl-CoAs into the mitochondria (Figure 1) (30). Thus, in the presence of abundant glucose, malonyl-CoA accumulates and fatty acid oxidation is inhibited. Malonyl-CoA generation has been implicated in glucose sensing in the liver to regulate ketogenesis (31) and the hypothalamus to regulate nutrient intake $(32,33)$.

$K_{A T P}$ channels. $\mathrm{K}_{\mathrm{ATP}}$ channel closure-induced membrane depolarization as a mechanism for glucose sensing is not reserved only for the pancreatic $\beta$ cell. Glucose-sensing hypothalamic neurons were initially identified in the 1960s based upon discrete sets of neurons that could be either electrically inhibited or excited by 
elevations in extracellular glucose (34). Neuronal glucose sensing has long been implicated in the regulation of autonomic nervous system-mediated changes in glucose homeostasis, particularly the counterregulatory response to hypoglycemia $(35,36)$. Seino and colleagues demonstrated that absence of functional $\mathrm{K}_{\mathrm{ATP}}$ channels as a result of knockout of the Kir6.2 subunit of the channel complex abolishes the glucose responsiveness of ventromedial hypothalamic neurons and is associated with loss of glucagon secretion in response to neuroglycopenia (37). While $\mathrm{K}_{\mathrm{ATP}}$-mediated glucose sensing appears operative in some glucose-responsive neurons, not all glucose-responsive neurons require $\mathrm{K}_{\mathrm{ATP}}$ channels for glucose sensing. For instance, Kir6.2 deficiency does not abolish glucose-induced excitation of glucose-sensing neurons in the arcuate nucleus (38). Recently, Rossetti and colleagues showed that in the presence of basal (low physiologic) insulin levels, icv infusion of glucose in rats directly suppresses endogenous glucose production without any detectable changes in glucoregulatory hormones (39). The suppressive effect of intracerebral glucose on endogenous glucose production could be inhibited with icv administration of the $\mathrm{K}_{\mathrm{ATP}}$ channel blocker glibenclamide, suggesting the same glucose sensor that plays a pivotal role in glucose homeostasis in the $\beta$ cell may play a similar role in the hypothalamus (39).

$\mathrm{K}_{\text {ATP }}$ channels sense glucose via changes in cellular energy status, but they are not the exclusive cellular mechanism for sensing energy status. AMP-activated protein kinase (AMPK) is a ubiquitous, evolutionarily conserved sensor of cellular energy status that is activated by increases in the cellular AMP/ATP ratio (40) (Figure 1). AMPK, when activated, phosphorylates a diverse set of enzymes and alters expression of numerous genes which result in increased energy availability and inhibition of energy-consuming processes within the cell. In certain tissues and situations, AMPK may also act as a glucose sensor. The energetic stress (e.g., ATP depletion) imposed by exercise in skeletal muscle leads to AMPK activation, which promotes fatty acid oxidation (41) and may contribute to increased insulin-independent glucose uptake (Figures 1 and 2), though this remains controversial (42). Recently, AMPK activity has been implicated in glucose sensing in the hypothalamus and in mediating the counterregulatory response to insulin-induced hypoglycemia (Figure 2) $(43,44)$.

Sirtuin 1 . The redox status of a cell, reflected by the lactate/pyruvate ratio, may be regulated independently of the energy status (45). Sirtuin 1 (SIRT1), the mammalian homolog of the NAD ${ }^{+}$dependent histone deacetylase Sir2, which regulates lifespan in Saccharomyces cerevisiae and Caenorhabditis elegans in response to caloric intake, has recently been shown to play a key role in regulating gluconeogenic and glycolytic pathways in mouse liver. Treatment of primary hepatocytes with pyruvate increased, and glucose decreased, protein levels of SIRT1 (46) (Figure 1). The mechanism mediating glucose/pyruvate sensing is uncertain, though the resulting change in SIRT1 protein levels is posttranscriptional. The combination of increased SIRT1 protein levels along with increased $\mathrm{NAD}^{+}$accumulation in fasted livers stimulates the SIRT1-mediated deacetylation of PPAR $\gamma$, coactivator $1-\alpha$ (PGC- $1 \alpha)$, which increases expression of gluconeogenic enzymes specifically, without affecting PGC-1 $\alpha$-regulated mitochondrial genes (46).

The transcriptional changes mediated by the SIRT1/PGC- $1 \alpha$ pathway may impact hepatic glucose production over the medium to long term, but the liver can also autoregulate its glucose production in response to a glucose load in the short term (reviewed in ref. 47). For instance, hyperglycemia causes a $60 \%$ reduction in net hepatic glucose output within 30 minutes in the conscious 36-hourfasted dog in the setting of basal levels of insulin and glucagon (48, 49). Hepatic glucose output is determined by the rates of net glycogenolysis, gluconeogenesis, and glucose cycling (the futile cycling between glucose and glucose-6-phosphate). It appears that all 3 of these parameters may change to restore euglycemia as a direct result of changes in ambient glucose levels (50). After short-term fasting, when glycogen stores are sufficient, hyperglycemia appears to reduce glucose production predominantly by decreasing the glycogenolytic rate and increasing glucose cycling. Glucose-6-phosphate, the first glucose metabolite in glycolysis, may account for some of these effects, since it is a potent allosteric activator of glycogen synthase that catalyzes glycogen synthesis (Figure 1) (51). In this case, glucose-6-phosphate resulting from glucose metabolism is the signal reporting glucose flux, while glycogen synthase is the sensor.

Carbobydrate response element-binding protein. Other glucose metabolites have also been suggested to have signaling roles. Carbohydrate response element-binding protein (ChREBP) is a transcription factor that mediates glucose-responsive changes in gene expression in liver and possibly other tissues (52). In the presence of glucose, ChREBP translocates from the cytosol to the nucleus, and its DNA binding/transcription activity is additionally stimulated, resulting in transcription of glycolytic and lipogenic enzymes. It has been proposed that xylulose-5-phosphate, a metabolite of glucose generated via the pentose phosphate pathway, activates a specific isoform of protein phosphatase $2 \mathrm{~A}$, which dephosphorylates ChREBP, contributing to its translocation and activation (53) (Figure 1).

Hexosamines. In addition, glucose metabolites in the hexosamine biosynthetic pathway have also been implicated as cellular signaling molecules. This pathway metabolizes glucose to uridine diphospho- $N$-acetyl glucosamine (UDP-GlcNAc), which is used in the synthesis of glycosaminoglycans, proteoglycans, and glycolipids. The amidation of fructose- 6 -phosphate to glucosamine-6-phosphate is the rate-limiting step in the pathway and is catalyzed by glutamine:fructose-6-phosphate amidotransferase (GFAT) (54). GFAT activity correlates with insulin sensitivity and adiposity (55). Transgenic overexpression of GFAT in mouse liver results in hyperlipidemia, obesity, and impaired glucose tolerance (56). Combined overexpression of GFAT in muscle and adipose tissue results in hyperleptinemia and insulin resistance, though explanted muscle from these mice did not demonstrate insulin resistance ex vivo (57). Adipose-specific overexpression of GFAT causes impaired glucose tolerance and skeletal muscle insulin resistance, again demonstrating that genetic modifications in adipose tissue may impact whole-body glucose homeostasis (58). Interestingly, in muscle-specific GLUT1 overexpressors, where increased glucose flux into muscle is constitutive, GFAT activity and the concentration of UDP-hexoses are increased. In contrast, neither GFAT activity nor UDP-hexose concentrations are increased in adipose/muscle-specific GLUT4 overexpressors (59). The differences in hexosamine production may account for the fact that insulin resistance develops in the GLUT1 overexpressors but not in the GLUT4 overexpressors.

Hepatoportal sensor. Hepatoportal glucose sensing (Figure 2) describes a well-characterized phenomenon by which a glucose gradient established between the portal vein and the hepatic artery is sensed, resulting in increased hepatic glucose uptake, increased peripheral glucose disposal, inhibition of counterregulatory hormone secretion, and inhibition of food intake leading to hypo- 


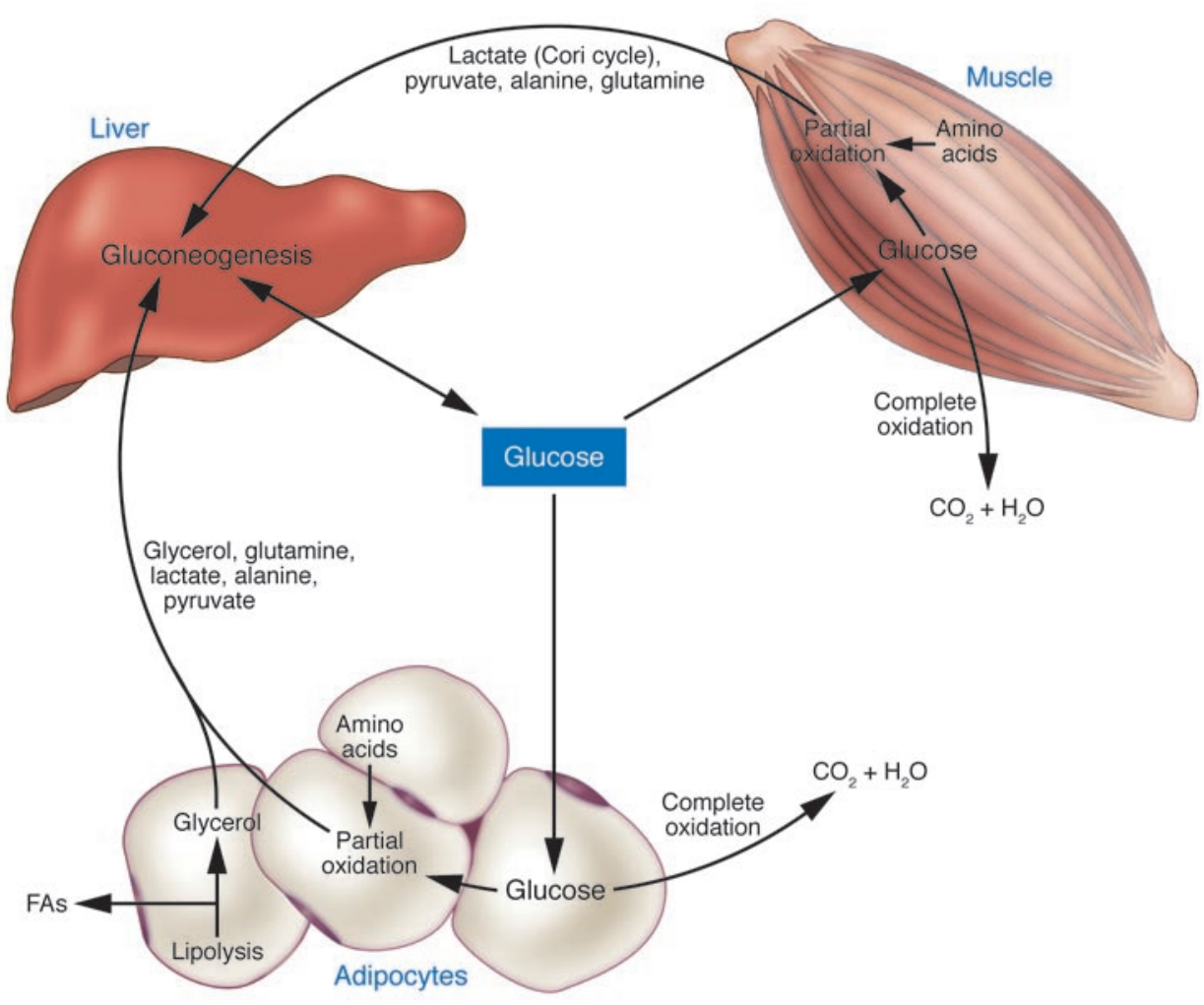

\section{Figure 3}

Substrate cycles as glucose-derived signals. Carbon that enters muscle and adipose tissue as glucose may be completely oxidized and leave the tissue as $\mathrm{CO}_{2}$ and $\mathrm{H}_{2} \mathrm{O}$. However, a substantial portion of the carbon that enters these tissues as glucose is recycled back to the liver in the form of various gluconeogenic precursors. These gluconeogenic precursors constitute signals reflecting the intracellular milieu in muscle and adipose tissue, which is determined by the physiologic state of the organism. Amino acids derived from protein breakdown that occurs predominantly in muscle provide nitrogen and carbon for the production of alanine and glutamine in muscle and adipose tissue. glycemia $(60,61)$. The actions of this sensor appear to depend on autonomic afferents to the CNS $(62,63)$. However, the precise cellular composition of this sensor and the mechanism by which it senses and communicates remain uncertain.

\section{Carbon skeleton cycling: a form of integrated glucose sensing?}

To understand how integrated glucose homeostasis is so precisely regulated by independent organs, each organ responding in its own way to hormonal and neural cues, we must develop a better appreciation for the complex interorgan fluxes of glucose in addition to the fluxes of circulating carbon substrates that can be converted into glucose (Figure 3). After glucose enters a specific tissue, its metabolic fate varies depending on the type of tissue and the cellular status of the tissue. Glucose can potentially be oxidized completely to $\mathrm{CO}_{2}$ and $\mathrm{H}_{2} \mathrm{O}$ or incorporated into glycogen or triglyceride for energy storage. Glucose may be used anabolically for de novo fatty acid synthesis or the synthesis of nonessential amino acids or other macromolecules. Glucose may be partially oxidized to lactate, which may return to the liver for gluconeogenesis, constituting the Cori cycle (Figure 3) (64). Additionally, glucose taken up into muscle may be converted to alanine, which serves (a) as a major gluconeogenic substrate constituting the glucose-alanine cycle; and (b) as a transporter of nitrogen generated by protein breakdown back to the liver for excretion as urea $(65,66)$. These and other exported glucose-derived metabolites serve as signals to other organs and may convey information reflective of the cellular status of their tissue of origin.

The complex cellular mechanisms that determine the partitioning of glucose metabolites inside a cell toward complete oxidation versus conversion to potential anabolic metabolites or recyclable carbon skeletons may in part be determined by regulation of the pyruvate dehydrogenase enzyme complex (67). Pyruvate dehydrogenase acts as the gatekeeper for pyruvate entry into the TCA cycle as acetyl-CoA. It is precisely regulated by a specific family of kinases as well as specific phosphatases in addition to complex allosteric regulation by additional metabolites (68-71). Thus, potential gluconeogenic carbon skeleton intermediates like lactate or alanine released from a tissue reflect the intracellular milieu of the tissue of origin and may therefore also be considered as signals communicating the cellular status of the tissue of origin. For instance, during intense exercise, lactate production as a result of intramuscular hypoxia may serve as a signal to increase endogenous glucose production. Increased lactate production by muscle would provide gluconeogenic substrate for the liver and might also alter the liver redox status, thereby regulating SIRT1 protein levels and Sirt1PGC1 $\alpha$ actions on gluconeogenesis (Figure 1). Increased lactate levels in the hypothalamus may also alter hepatic glucose production through neural pathways (39). In addition, the acidosis accompanying lactate production as a result of exercise has been implicated as a stimulus to increase renal gluconeogenesis $(72,73)$. Thus, glucose-derived metabolites, released from tissues where glucose is taken up, may act as signals in distant organs (Figure 3).

\section{The role of adipocytes in integrated fuel homeostasis}

The whole-body insulin resistance caused by adipose-specific knockout of GLUT4 (74) underscores how the adipocyte affects systemic glucose homeostasis as a direct result of limiting glucose flux into the adipocyte. Adipocytes are increasingly recognized as sources of circulating hormones, cytokines, and substrates that can influence systemic energy balance and glucose homeostasis (Figure 4) (75). Several of these hormones that have been well studied are leptin, adiponectin, and resistin (76-82). Since the secretion of these hormones is altered in insulin-resistant states and GLUT4 


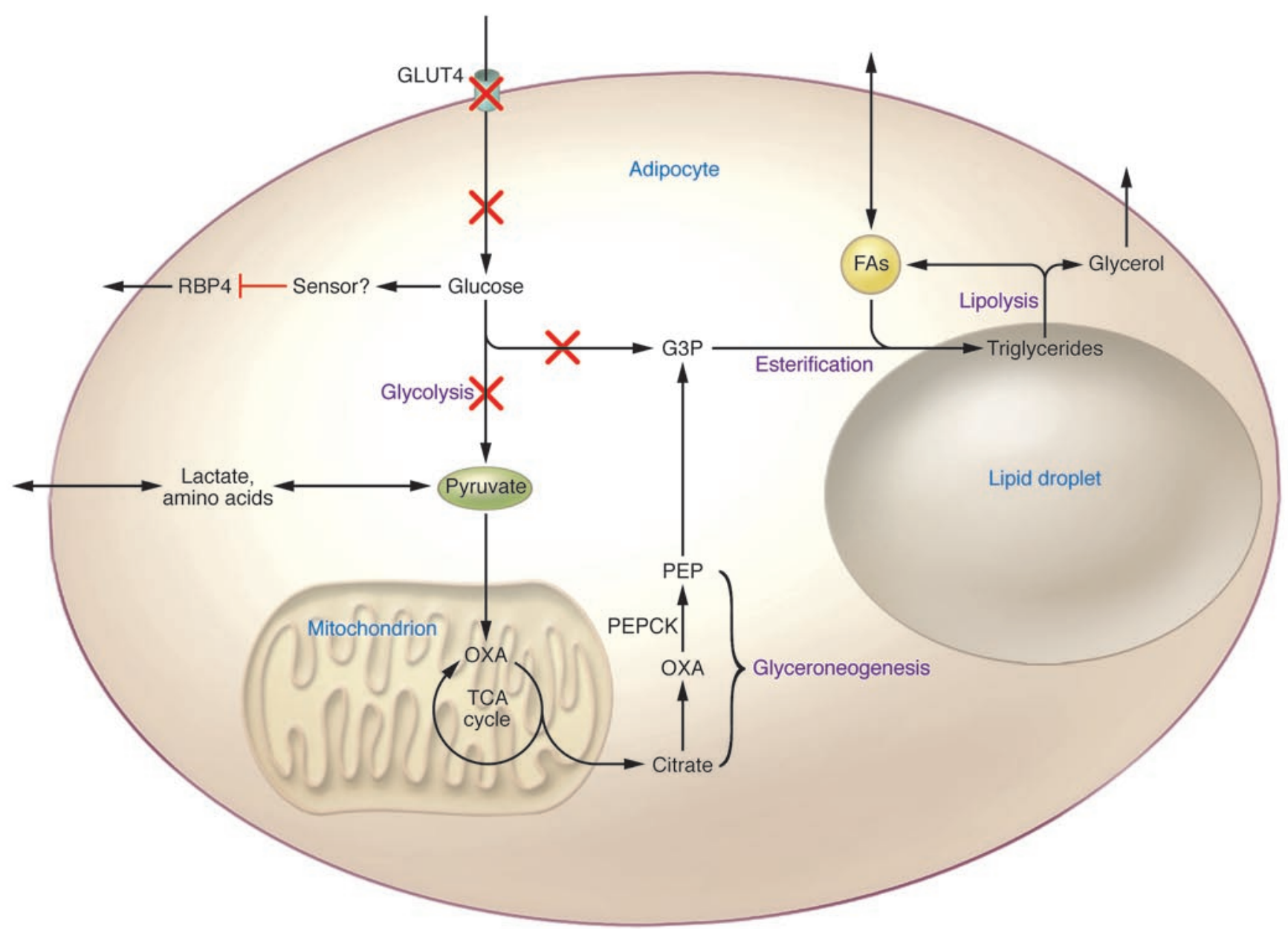

Figure 4

The adipocyte as a glucose sensor. Physiologic downregulation of GLUT4 in the fasted state, pathologic downregulation in insulin-resistant states, or genetic knockout result in diminished glucose flux. The diminished glucose flux is sensed by the adipocyte, resulting in increased RBP4 secretion. Additionally, the diminished glucose flux may limit the ability to generate glycerol-3-phosphate via glycolysis. The adipocyte becomes reliant on glyceroneogenesis for glycerol-3-phosphate production, which may be limiting for fatty acid re-esterification and contribute to increased fatty acid release. G3P, glycerol-3-phosphate; PEP, phosphoenolpyruvate.

is downregulated in adipose tissue in insulin-resistant humans and rodents (3), the potential role of glucose transport in regulating the expression or secretion of adipokines has been studied, though these studies are inconclusive $(83,84)$.

GLUT4 is physiologically downregulated specifically in adipose tissue in fasting conditions, and it is rapidly upregulated following refeeding (85). The seeming paradox that GLUT4 is normally downregulated in fasting but is also downregulated in obesity (a state of nutritional excess) leads us to ask, Does the adipocyte sense a signal that causes GLUT4 downregulation in states of both nutrient depletion and chronic excess? How is the diminished glucose flux sensed, and how is this signal communicated to the rest of the body?

In the AG4KO mouse, no alterations in serum levels of the classical adipokines have been identified (74). Thus, in this model, adipose tissue must communicate by altering some other hormone, neural pathway, or substrate. As mentioned above, we recently identified RBP4 as a new adipokine that contributes to insulin resistance in the AG4KO mouse. The mechanism by which diminished glucose flux is translated into increased RBP4 secretion remains under investigation. However, even in normal mice, pharmacologically or genetically increasing serum RBP4 levels impairs insulin signaling in muscle and increases phosphoenolpyruvate by inducing cytosolic phosphoenolpyruvate carboxykinase (PEPCK) expression in liver, resulting in systemic insulin resistance (20). Interestingly, serum RBP4 levels are elevated in insulin-resistant obese and type 2 diabetic people, and the magnitude of the elevation correlates highly with the degree of insulin resistance and with multiple features of the metabolic syndrome (86). RBP4 elevation precedes overt diabetes and may serve as a clinically useful marker (86). Additional means of communication may be employed by adipocytes when GLUT4 expression is downregulated.

The adipocyte is the primary storage depot for triglycerides, and perturbed fatty acid homeostasis has been implicated in causing insulin resistance and type 2 diabetes. Fatty acid release from adipocytes is determined by the rates of lipolysis and fatty acid re-esterification. Glycerol-3-phosphate is required for fatty acid esterification, and its availability may play a critical role in regulating lipolysis. In normal individuals in the fed state, glycerol-3- 
phosphate is generated via glycolysis (Figure 4). However, in the fasted state, when glucose flux is diminished due to low insulin levels and decreased GLUT4 expression, glycerol-3-phosphate must be generated by alternative means. Whereas liver can reuse glycerol hydrolyzed from triglycerides to produce glycerol-3-phosphate via the enzyme glycerol kinase, adipose tissue contains relatively low amounts of this enzyme. In adipose tissue in the fasted state, glycerol-3-phosphate is generated by an abbreviated version of gluconeogenesis termed glyceroneogenesis (Figure 4) (87). In this pathway, anaplerotic metabolites such as lactate or pyruvate enter the TCA cycle, exit to the cytosol as oxaloacetate, and are then converted to phosphoenolpyruvate by cytosolic PEPCK, the rate-limiting step in gluconeogenesis in kidney and liver. Phosphoenolpyruvate can then be converted to glycerol-3-phosphate by reverse glycolysis. Glyceroneogenesis is a major pathway for glycerol-3-phosphate production and fatty acid esterification in adipocytes in mice fed a low-carbohydrate diet studied in vivo and in human subcutaneous adipose tissue explants studied ex vivo $(88,89)$. Interestingly, thiazolidinediones (TZDs), potent insulin-sensitizing drugs, upregulate both glycerol kinase and PEPCK activity in adipose tissue, which may result in retention of fatty acids through re-esterification, limiting the release of free fatty acids into the circulation $(88,89)$. This re-esterification may contribute to the weight gain commonly encountered in patients treated with TZDs. Thus, the reduction of glucose flux into adipose tissue that occurs in insulin-resistant states would result in a reliance on glyceroneogenesis and glycerol kinase for glycerol-3phosphate production. In the setting of elevated fatty acid turnover, these alternative pathways could potentially be saturated, and the subsequent release of fatty acids could be considered a signal of relative glucose deficiency in the adipocyte. While plasma free fatty acid levels are not elevated in the AG4KO mouse compared with control animals (74), the rates of fatty acid esterification and turnover have not been directly assessed.

\section{Summary}

Transgenic overexpression or knockout of GLUT1 or GLUT4 in specific tissues has led to unexpected findings, including the fact that tissues such as muscle and adipose can sense glucose and communicate changes in glucose flux to other tissues. In addition, glucose and its metabolites act as signaling molecules. Cellular sensors that respond to these signals are being identified. The metabolic fate of glucose and the signals that are generated depend on tissue-specific factors and the cellular status of the tissue, which is highly affected by the nutritional and energetic state of the organism. Metabolism of glucose and the accompanying signals that are generated alter the availability of other fuels such as fatty acids that are also thought to play a major role in the pathogenesis of type 2 diabetes. Exciting advances are taking place in understanding how different tissues generate these signals and which sensing pathways are critical for maintaining metabolic harmony.

\section{Acknowledgments}

The authors thank Timothy Graham for stimulating discussions that helped to shape the concepts discussed and members of the Kahn laboratory for invaluable contributions to the ongoing work. Work in the authors' laboratory is supported by NIH grants R01 DK43051, R01 DK60839, P01 DK56116, P30 DK57521, and T32 DK07516.

Address correspondence to: Barbara B. Kahn, Division of Endocrinology, Diabetes and Metabolism, Beth Israel Deaconess Medical Center, 99 Brookline Avenue, Boston, Massachusetts 02215, USA. Phone: (617) 667-5422; Fax: (617) 667-2927; E-mail: bkahn@ bidmc.harvard.edu.
1. Mokdad, A.H., et al. 2003. Prevalence of obesity, diabetes, and obesity-related health risk factors, 2001. JAMA. 289:76-79.

2. McGarry, J.D. 1992. What if Minkowski had been ageusic? An alternative angle on diabetes. Science. 258:766-770.

3. Shepherd, P.R., and Kahn, B.B. 1999. Glucose transporters and insulin action - implications for insulin resistance and diabetes mellitus. N. Engl. J. Med. 341:248-257.

4. DeFronzo, R. 1997. Pathogenesis of type 2 diabetes: metabolic and molecular implications for identifying diabetes genes. Diabetes Reviews. 5:177-269.

5. Storlien, L., Oakes, N.D., and Kelley, D.E. 2004. Metabolic flexibility. Proc. Nutr. Soc. 63:363-368.

6. Kelley, D.E., Goodpaster, B., Wing, R.R., and Simoneau, J.A. 1999. Skeletal muscle fatty acid metabolism in association with insulin resistance, obesity, and weight loss. Am. J. Physiol. 277:E1130-E1141.

7. Kahn, B.B., and Rossetti, L. 1998. Type 2 diabetes - who is conducting the orchestra? Nat. Genet. 20:223-225.

8. Wasserman, D.H. 1995. Regulation of glucose fluxes during exercise in the postabsorptive state. Annu. Rev. Physiol. 57:191-218.

9. Joost, H.G., et al. 2002. Nomenclature of the GLUT/SLC2A family of sugar/polyol transport facilitators. Am. J. Physiol. Endocrinol. Metab. 282:E974-E976.

10. Marshall, B.A., et al. 1993. Germline manipulation of glucose homeostasis via alteration of glucose transporter levels in skeletal muscle. J. Biol. Chem. 268:18442-18445.

11. Marshall, B.A., and Mueckler, M.M. 1994. Differential effects of GLUT-1 or GLUT-4 overexpression on insulin responsiveness in transgenic mice. Am.J. Physiol. 267:E738-E744.

12. Liu, M.L., et al. 1993. Transgenic mice expressing the human GLUT4/muscle-fat facilitative glucose transporter protein exhibit efficient glycemic control. Proc. Natl. Acad. Sci. U. S. A. 90:11346-11350.

13. Leturque, A., Loizeau, M., Vaulont, S., Salminen, M., and Girard, J. 1996. Improvement of insulin action in diabetic transgenic mice selectively overexpressing GLUT4 in skeletal muscle. Diabetes. 45:23-27.

14. Tsao, T.S., Burcelin, R., Katz, E.B., Huang, L., and Charron, M.J. 1996. Enhanced insulin action due to targeted GLUT4 overexpression exclusively in muscle. Diabetes. 45:28-36.

15. Tozzo, E., Shepherd, P.R., Gnudi, L., and Kahn, B.B. 1995. Transgenic GLUT-4 overexpression in fat enhances glucose metabolism: preferential effect on fatty acid synthesis. Am. J. Physiol. 268:E956-E964.

16. Carvalho, E., Kotani, K., Peroni, O.D., and Kahn, B.B. 2005. Adipose-specific overexpression of GLUT4 reverses insulin resistance and diabetes in mice lacking GLUT4 selectively in muscle. Am. J. Physiol. Endocrinol. Metab. 289:E551-E561.

17. Zisman, A., et al. 2000. Targeted disruption of the glucose transporter 4 selectively in muscle causes insulin resistance and glucose intolerance. Nat. Med. 6:924-928.

18. Kim, J.K., et al. 2001. Glucose toxicity and the development of diabetes in mice with muscle-specific inactivation of GLUT4. J. Clin. Invest. 108:153-160. doi:10.1172/JCI200110294.

19. Kraegen, E.W., James, D.E., Jenkins, A.B., and Chisholm, D.J. 1985. Dose-response curves for in vivo insulin sensitivity in individual tissues in rats. Am. J. Physiol. 248:E353-E362.

20. Yang, Q., et al. 2005. Serum retinol binding protein 4 contributes to insulin resistance in obesity and type 2 diabetes. Nature. 436:356-362.

21. Chevalier, S., Marliss, E.B., Morais, J.A., Lamarche, M., and Gougeon, R. 2005. Whole-body protein anabolic response is resistant to the action of insulin in obese women. Am. J. Clin. Nutr. 82:355-365.

22. Dennis, P.B., et al. 2001. Mammalian TOR: a homeostatic ATP sensor. Science. 294:1102-1105.

23. Nobukuni, T., et al. 2005. Amino acids mediate $\mathrm{mTOR} /$ raptor signaling through activation of class 3 phosphatidylinositol 3OH-kinase. Proc. Natl. Acad. Sci. U. S. A. 102:14238-14243.

24. Matschinsky, F.M., Glaser, B., and Magnuson, M.A. 1998. Pancreatic beta-cell glucokinase: closing the gap between theoretical concepts and experimental realities. Diabetes. 47:307-315.

25. Schuit, F.C., Huypens, P., Heimberg, H., and Pipeleers, D.G. 2001. Glucose sensing in pancreatic beta-cells: a model for the study of other glucoseregulated cells in gut, pancreas, and hypothalamus. Diabetes. 50:1-11.

26. Schuit, F., et al. 1997. Metabolic fate of glucose in purified islet cells. Glucose-regulated anaplerosis in beta cells. J. Biol. Chem. 272:18572-18579.

27. Owen, O.E., Kalhan, S.C., and Hanson, R.W. 2002. The key role of anaplerosis and cataplerosis for citric acid cycle function. J. Biol. Chem. 277:30409-30412.

28. MacDonald, M.J., et al. 2005. Perspective: emerging evidence for signaling roles of mitochondrial anaplerotic products in insulin secretion. Am.J. Physiol. Endocrinol. Metab. 288:E1-E15.

29. Ruderman, N.B., Saha, A.K., Vavvas, D., and Wit- 
ters, L.A. 1999. Malonyl-CoA, fuel sensing, and insulin resistance. Am. J. Physiol. 276:E1-E18.

30. McGarry, J.D., Mills, S.E., Long, C.S., and Foster, D.W. 1983. Observations on the affinity for carnitine, and malonyl-CoA sensitivity, of carnitine palmitoyltransferase I in animal and human tissues. Demonstration of the presence of malonyl$\mathrm{CoA}$ in non-hepatic tissues of the rat. Biochem. $J$. 214:21-28.

31. McGarry, J.D., Mannaerts, G.P., and Foster, D.W. 1977. A possible role for malonyl-CoA in the regulation of hepatic fatty acid oxidation and ketogenesis. J. Clin. Invest. 60:265-270.

32. He, W., Lam, T.K., Obici, S., and Rossetti, L. 2006. Molecular disruption of hypothalamic nutrient sensing induces obesity. Nat. Neurosci. 9:227-233.

33. Hu, Z., Dai, Y., Prentki, M., Chohnan, S., and Lane, M.D. 2005. A role for hypothalamic malonylCoA in the control of food intake. J. Biol. Chem. 280:39681-39683.

34. Oomura, Y., Ono, T., Ooyama, H., and Wayner, M.J. 1969. Glucose and osmosensitive neurones of the rat hypothalamus. Nature. 222:282-284.

35. Cryer, P.E., Davis, S.N., and Shamoon, H. 2003. Hypoglycemia in diabetes. Diabetes Care. 26:1902-1912.

36. Havel, P.J., and Taborsky, G.J., Jr. 1989. The contribution of the autonomic nervous system to changes of glucagon and insulin secretion during hypoglycemic stress. Endocr. Rev. 10:332-350.

37. Miki, T., et al. 2001. ATP-sensitive $\mathrm{K}+$ channels in the hypothalamus are essential for the maintenance of glucose homeostasis. Nat. Neurosci. 4:507-512.

38. Fioramonti, X., Lorsignol, A., Taupignon, A., and Penicaud, L. 2004. A new ATP-sensitive K+ channel-independent mechanism is involved in glucoseexcited neurons of mouse arcuate nucleus. Diabetes. 53:2767-2775.

39. Lam, T.K., Gutierrez-Juarez, R., Pocai, A., and Rossetti, L. 2005. Regulation of blood glucose by hypothalamic pyruvate metabolism. Science. 309:943-947.

40. Kahn, B.B., Alquier, T., Carling, D., and Hardie, D.G. 2005. AMP-activated protein kinase: ancient energy gauge provides clues to modern understanding of metabolism. Cell Metab. 1:15-25.

41. Merrill, G.F., Kurth, E.J., Hardie, D.G., and Winder, W.W. 1997. AICA riboside increases AMPactivated protein kinase, fatty acid oxidation, and glucose uptake in rat muscle. Am. J. Physiol. 273:E1107-E1112.

42. Fujii, N., et al. 2005. AMP-activated protein kinase alpha2 activity is not essential for contraction- and hyperosmolarity-induced glucose transport in skeletal muscle. J. Biol. Chem. 280:39033-39041.

43. Minokoshi, Y., et al. 2004. AMP-kinase regulates food intake by responding to hormonal and nutrient signals in the hypothalamus. Nature. 428:569-574.

44. Han, S.M., et al. 2005. Hypothalamic AMP-activated protein kinase mediates counter-regulatory responses to hypoglycaemia in rats. Diabetologia. 48:2170-2178.

45. Sistare, F.D., and Haynes, R.C., Jr. 1985. The interaction between the cytosolic pyridine nucleotide redox potential and gluconeogenesis from lactate/ pyruvate in isolated rat hepatocytes. Implications for investigations of hormone action. J. Biol. Chem. 260:12748-12753.

46. Rodgers, J.T., et al. 2005. Nutrient control of glucose homeostasis through a complex of PGC1alpha and SIRT1. Nature. 434:113-118.

47. Moore, M.C., Connolly, C.C., and Cherrington, A.D. 1998. Autoregulation of hepatic glucose production. Eur. J. Endocrinol. 138:240-248.

48. Shulman, G.I., Liljenquist, J.E., Williams, P.E., and Lacy, W.W. 1978. Glucose disposal during insulinopenia in somatostatin-treated dogs. The roles of glucose and glucagon. J. Clin. Invest. 62:487-491.

49. Shulman, G.I., et al. 1980. Effect of glucose, inde- pendent of changes in insulin and glucagon secretion, on alanine metabolism in the conscious dog. J. Clin. Invest. 65:496-505.

50. Rossetti, L., et al. 1993. Mechanism by which hyperglycemia inhibits hepatic glucose production in conscious rats. Implications for the pathophysiology of fasting hyperglycemia in diabetes. J. Clin. Invest. 92:1126-1134

51. Shulman, R.G., Bloch, G., and Rothman, D.L. 1995. In vivo regulation of muscle glycogen synthase and the control of glycogen synthesis. Proc. Natl. Acad. Sci. U. S. A. 92:8535-8542.

52. Yamashita, H., et al. 2001. A glucose-responsive transcription factor that regulates carbohydrate metabolism in the liver. Proc. Natl. Acad. Sci. U. S. A. 98:9116-9121.

53. Kabashima, T., Kawaguchi, T., Wadzinski, B.E., and Uyeda, K. 2003. Xylulose 5-phosphate mediates glucose-induced lipogenesis by xylulose 5 -phosphate-activated protein phosphatase in rat liver. Proc. Natl. Acad. Sci. U. S. A. 100:5107-5112.

54. McKnight, G.L., et al. 1992. Molecular cloning, cDNA sequence, and bacterial expression of human glutamine:fructose-6-phosphate amidotransferase. J. Biol. Chem. 267:25208-25212.

55. Daniels, M.C., Ciaraldi, T.P., Nikoulina, S., Henry, R.R., and McClain, D.A. 1996. Glutamine:fructose6-phosphate amidotransferase activity in cultured human skeletal muscle cells: relationship to glucose disposal rate in control and non-insulin-dependent diabetes mellitus subjects and regulation by glucose and insulin. J. Clin. Invest. 97:1235-1241.

56. Veerababu, G., et al. 2000. Overexpression of glutamine:fructose-6-phosphate amidotransferase in the liver of transgenic mice results in enhanced glycogen storage, hyperlipidemia, obesity, and impaired glucose tolerance. Diabetes. 49:2070-2078.

57. Hebert, L.F., Jr., et al. 1996. Overexpression of glutamine:fructose-6-phosphate amidotransferase in transgenic mice leads to insulin resistance. J. Clin. Invest. 98:930-936.

58. Hazel, M., et al. 2004. Activation of the hexosamine signaling pathway in adipose tissue results in decreased serum adiponectin and skeletal muscle insulin resistance. Endocrinology. 145:2118-2128.

59. Buse, M.G., Robinson, K.A., Marshall, B.A., and Mueckler, M. 1996. Differential effects of GLUT1 or GLUT4 overexpression on hexosamine biosynthesis by muscles of transgenic mice. J. Biol. Chem. 271:23197-23202.

60. Burcelin, R., Dolci, W., and Thorens, B. 2000. Portal glucose infusion in the mouse induces hypoglycemia: evidence that the hepatoportal glucose sensor stimulates glucose utilization. Diabetes. 49:1635-1642.

61. Hevener, A.L., Bergman, R.N., and Donovan, C.M. 1997. Novel glucosensor for hypoglycemic detection localized to the portal vein. Diabetes. 46:1521-1525.

62. Fujita, S., and Donovan, C.M. 2005. Celiac-superior mesenteric ganglionectomy, but not vagotomy, suppresses the sympathoadrenal response to insulin-induced hypoglycemia. Diabetes. 54:3258-3264.

63. Cardin, S., et al. 2001. Effect of vagal cooling on the counterregulatory response to hypoglycemia induced by a low dose of insulin in the conscious dog. Diabetes. 50:558-564.

64. Katz, J., and Tayek, J.A. 1998. Gluconeogenesis and the Cori cycle in 12-, 20-, and 40-h-fasted humans. Am. J. Physiol. 275:E537-E542.

65. Perriello, G., et al. 1995. Estimation of glucosealanine-lactate-glutamine cycles in postabsorptive humans: role of skeletal muscle. Am. J. Physiol. 269:E443-E450.

66. Felig, P. 1973. The glucose-alanine cycle. Metabolism. 22:179-207.

67. Randle, P.J. 1986. Fuel selection in animals. Biochem. Soc. Trans. 14:799-806.

68. Huang, B., et al. 1998. Isoenzymes of pyruvate dehydrogenase phosphatase. DNA-derived amino acid sequences, expression, and regulation. J. Biol. Chem. 273:17680-17688.

69. Bowker-Kinley, M.M., Davis, W.I., Wu, P., Harris, R.A., and Popov, K.M. 1998. Evidence for existence of tissue-specific regulation of the mammalian pyruvate dehydrogenase complex. Biochem. J. 329:191-196.

70. Wieland, O.H. 1983. The mammalian pyruvate dehydrogenase complex: structure and regulation. Rev. Physiol. Biochem. Pharmacol. 96:123-170.

71. Wu, P., et al. 1998. Starvation and diabetes increase the amount of pyruvate dehydrogenase kinase isoenzyme 4 in rat heart. Biochem. J. 329:197-201.

72. Hortelano, P., et al. 1984. Renal ammoniagenesis in rats made acutely acidotic by swimming. Horm. Metab. Res. 16:370-373.

73. Munoz-Clares, R., Garcia-Ruiz, J.P., Vargas, A., and Sanchez-Medina, F. 1979. Effect of short-term exercise on the content of gluconeogenic metabolites in rat liver and kidney. FEBS Lett. 99:340-342.

74. Abel, E.D., et al. 2001. Adipose-selective targeting of the GLUT4 gene impairs insulin action in muscle and liver. Nature. 409:729-733.

75. Kershaw, E.E., and Flier, J.S. 2004. Adipose tissue as an endocrine organ. J. Clin. Endocrinol. Metab. 89:2548-2556.

76. Ahima, R.S., et al. 1996. Role of leptin in the neuroendocrine response to fasting. Nature. 382:250-252.

77. Friedman, J.M., and Halaas, J.L. 1998. Leptin and the regulation of body weight in mammals. Nature. 395:763-770.

78. Minokoshi, Y., et al. 2002. Leptin stimulates fattyacid oxidation by activating AMP-activated protein kinase. Nature. 415:339-343.

79. Yamauchi, T., et al. 2001. The fat-derived hormone adiponectin reverses insulin resistance associated with both lipoatrophy and obesity. Nat. Med. 7:941-946.

80. Yamauchi, T., et al. 2002. Adiponectin stimulates glucose utilization and fatty-acid oxidation by activating AMP-activated protein kinase. Nat. Med. 8:1288-1295.

81. Steppan, C.M., et al. 2001. The hormone resistin links obesity to diabetes. Nature. 409:307-312.

82. Rajala, M.W., Obici, S., Scherer, P.E., and Rossetti, L. 2003. Adipose-derived resistin and gut-derived resistin-like molecule- $\beta$ selectively impair insulin action on glucose production. J. Clin. Invest. 111:225-230. doi:10.1172/JCI200316521.

83. Cammisotto, P.G., Gelinas, Y., Deshaies, Y., and Bukowiecki, L.J. 2005. Regulation of leptin secretion from white adipocytes by insulin, glycolytic substrates, and amino acids. Am. J. Physiol. Endocrinol. Metab. 289:E166-E171.

84. Mueller, W.M., et al. 1998. Evidence that glucose metabolism regulates leptin secretion from cultured rat adipocytes. Endocrinology. 139:551-558.

85. Kahn, B.B., Cushman, S.W., and Flier, J.S. 1989. Regulation of glucose transporter-specific mRNA levels in rat adipose cells with fasting and refeeding. Implications for in vivo control of glucose transporter number. J. Clin. Invest. 83:199-204.

86. Graham, T.E., et al. 2006. Retinol binding protein-4 and insulin resistance in lean, obese, and type 2 diabetic subjects. N. Engl. J. Med. 354:22-33.

87. Reshef, L., Hanson, R.W., and Ballard, F.J. 1970. A possible physiological role for glyceroneogenesis in rat adipose tissue. J. Biol. Chem. 245:5979-5984.

88. Chen, J.L., et al. 2005. Physiologic and pharmacologic factors influencing glyceroneogenic contribution to triacylglyceride glycerol measured by mass isotopomer distribution analysis. J. Biol. Chem. 280:25396-25402.

89. Leroyer, S., et al. 2006. Rosiglitazone controls fatty acid cycling in human adipose tissue by means of glyceroneogenesis and glycerol phosphorylation. J. Biol. Chem. 281:13141-13149. 